\title{
Powerful surface-wave oscillators with one-dimensional and two- dimensional periodic planar structures
}

\author{
V.Yu. Zaslavsky, N.S. Ginzburg, A.M. Malkin, A.S. Sergeev \\ Institute of Applied Physics of the Russian Academy of Sciences, Niznhny Novgorod, Russia, zas-vladislav@ipfran.ru
}

Within the framework of a quasi-optical approach, we develop self-consistent theory of relativistic surface-wave oscillators [1]. Presenting the radiation field as a sum of two counter-propagating wavebeams coupled on a shallow corrugated surface, we describe formation of an evanescent slow wave. Dispersion characteristics of the evanescent wave following from this method are in good compliance with those found from the direct CST simulations. Considering excitation of the slow wave by a sheet electron beam, we simulate linear and nonlinear stages of interaction, which allows us to determine oscillation threshold conditions, electron efficiency and output coupling. The transition from the model of surfacewave oscillator operating in the $\pi$-mode regime to the canonical model of relativistic BWO is considered. We also described a modified scheme of planar relativistic surface-wave oscillators exploiting twodimensional periodic gratings [2]. Additional transverse propagating waves emerging on these gratings synchronize the emission from a wide sheet rectilinear electron beam allowing realization of a Cherenkov millimeter-wave oscillators with subgigawatt output power level.

\section{Simulations of surface-wave oscillators with con- ventional single periodic slow-wave structures in the framework of a 3D model}

We start with consideration of a surface-wave oscillator driven by a sheet electron beam with a finite width of $l_{x}^{e}$ over the $x$ axis (Fig. 1a). We should assume that the field structure is non-fixed over all three spatial coordinates. Thus, 3D quasi-optical model was used in simulations [3].

Results of simulations are depicted in Fig. 1 for the electron beam width of $l_{x}^{e}=10 \mathrm{~cm}$. At a relatively small width of the beam $l_{x}^{e}=5 \mathrm{~cm}$ the transverse (over $x$ ) field structure possesses a regular symmetric distribution (an exponential decay in the $y$-direction perpendicularly to the corrugated structure takes place in all variants). For wider beams, for example, for the electron beam width of $l_{x}^{e}=10 \mathrm{~cm}$, multistability regimes take place. For various initial conditions, we observed the excitation of both symmetrical and antisymmetrical modes, as shown in Fig. 1. For explanation we analyzed the dependence of temporal gain on beam width for symmetrical and anti-symmetrical modes. For rather small widths, gain for the symmetrical mode strongly exceeds gain for anti-symmetrical one. Under such conditions, a regular symmetric distribution sets on in the steady-state regime. With increasing beam width, gains for symmetrical and anti- symmetrical modes (and other modes) become very close, which leads to multistability.

Thus, diffraction of radiation in the $\mathrm{x}$-direction is sufficient for formation of regular field structure over this coordinate while the beam size is restricted by Fresnel condition, $N_{F}=l_{x}^{e 2} / 4 l_{z} \lambda \leq 1$. For practical estimations of the conventional scheme of a surfacewave oscillator we can take the beam width of 4-5 wavelengths, which corresponds to the regular field distribution. For a relatively high electron efficiency of about $10 \%$, the total output power amounts about $40 \mathrm{MW}$. For a metallic (brass) surface, Ohmic losses did not exceed several per cent of radiated power.

(a)

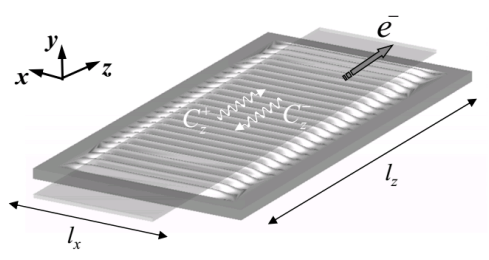

(b)

(c)
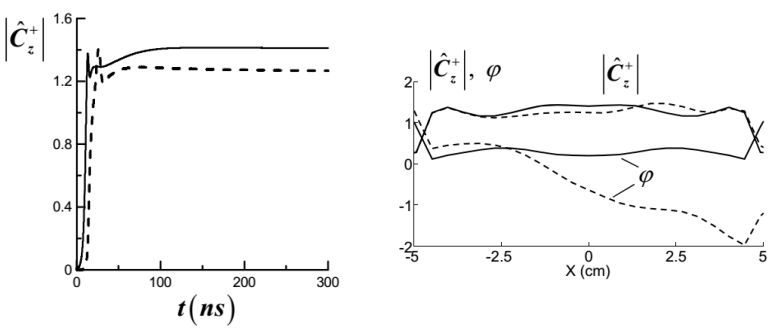

(d)

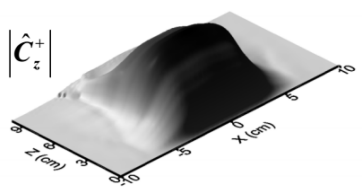

Fig. 1. (a) Scheme of surface-wave oscillator with 1D periodical gratings driven by a rectilinear sheet electron beam. Simulations of surface-wave oscillator based on 3D quasioptical model for electron beam width $l_{x}^{e}=10 \mathrm{~cm}$ :

(b) Temporal dependence of amplitude $\left|\hat{C}_{z}^{+}\right|$, (c) distributions of amplitude $\left|\hat{C}_{z}^{+}\right|$and phase $\varphi=\arg \left(\hat{C}_{z}^{+}\right)$at the output cross-section $z=l_{z} \quad\left(I_{0}=0.3 \mathrm{kA} / \mathrm{cm}, \quad \gamma_{0}=3\right.$, $l_{z}=10 \mathrm{~cm}$ ), (d) spatial distribution of this amplitude in the steady-state regime in the cross-section $y=$ const. Solid line corresponds to excitation of symmetrical mode, dashed line to anti-symmetrical one.

It should be noted that in 3D quasi-optical analysis we considered an idealized model of grating infinite in the transverse $x$ direction. On the basis of CST STUDIO SUITE software, we simulated a more realis- 
tic model of the grating with the same transverse width as the electron beam. In this case we found more severe restrictions on stability of singlefrequency oscillation regime. In fact, for electron beam and corrugation parameters indicated above, we observed single-frequency oscillation at the system width of up to 4-5 wavelengths. For larger width values, multifrequency generation regimes with simultaneous excitation of several modes with different numbers of variations over the $\mathrm{x}$ coordinate are more typical.

\section{Surface-Wave Oscillators with Two-Dimensional Periodic Structures}

In the case when the beam width substantially exceeds the wavelength, we suggest a more effective mechanism for provision of transverse radiation coherence based on the improved version of the slowwave structure, namely a double periodic grating $[2,3]$. Similarly to free electron masers (FEMs) with 2D distributed feedback, these gratings allow organizing additional transverse (with respect to the translational velocity of the electrons) electromagnetic energy fluxes that synchronize the radiation from wide sheet electron beams (Fig. 2).

Based on quasi-optical approach [2,3], we simulated the operation of a powerful $\mathrm{W}$-band relativistic surface-wave oscillator driven by a sheet beam with parameters close to those of the beam realized at a high-current ELMI accelerator (Budker Institute, Novosibirsk). We took the electron energy of $1 \mathrm{MeV}$, the beam width of $l_{x}=27 \mathrm{~cm}$, and the linear current density of $I_{0}=0.3 \mathrm{kA} / \mathrm{cm}$.

Onset of steady-state oscillations is shown in Fig. 2b. Temporal dependencies of energy fluxes radiated from different edges of the interaction space demonstrate that in the variant under consideration the most part of the power is radiated in the backward and forward directions (Fig. 2c). The power associated with transverse fluxes is relatively small. In accordance with the negative frequency, the shift from the Bragg frequency in the steady-state regime the partial wave fields are evanescent. In the $(z, x)$ plane the partial wave amplitudes possess bell-shaped profiles which are also similar to those found for the "cold" mode. Electron efficiency of about $10 \%$ at the chosen parameters corresponds to the integral radiation power of $\sim 0.9 \mathrm{GW}$. (a)

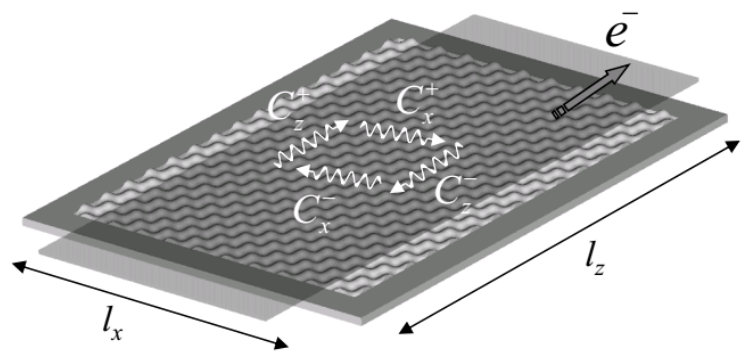

(b)

(c)
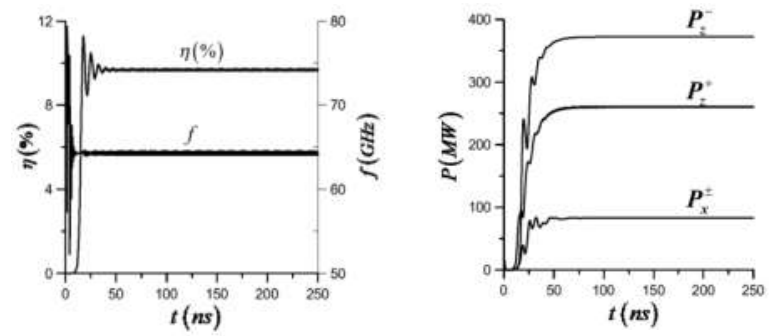

(d)

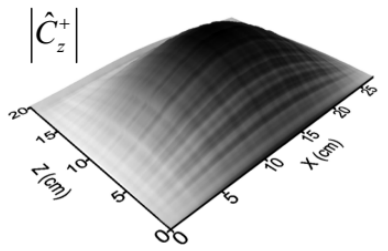

Fig. 2. (a) Scheme of surface-wave oscillator with 2D periodical grating. Temporal dependencies of (b) the efficiency and (c) the radiation power emitted in the different directions during the onset of a steady-state regime. (d) Spatial distribution of partial wave-beam $\left|\hat{C}_{z}^{+}\right|$in the steady-state regime $\left(I_{0} \approx 0.3 \mathrm{kA} / \mathrm{cm}, \gamma_{0}=3, l_{z}=19.6 \mathrm{~cm}, l_{x}=27 \mathrm{~cm}\right)$.

The work was supported by the Russian Science Foundation under grant No. 18-79-10252.

\section{References}

1. N.S. Ginzburg, A.M. Malkin, A.S. Sergeev, V.Yu. Zaslavsky. Quasi-optical theory of relativistic submillimeter surface-wave oscillators. Applied Physics Letters. 2011. V. 99. P. 121505.

2. Ginzburg N.S., Malkin A.M., Sergeev A.S., and Zaslavsky V.Yu. Powerful surface-wave oscillators with two-dimensional periodic structures. // Applied Physics Letters. 2012. V.100. P. 143510.

3. N.S. Ginzburg, A.M. Malkin, A.S. Sergeev, V.Yu. Zaslavsky. Quasi-Optical Theory of Relativistic Surface-Wave Oscillators with $1 \mathrm{D}$ and 2D Periodic Planar Structures. // Physics of Plasmas. 2013. V. 20, P. 113104. 